\title{
Readmission after discharge from a medium secure unit
}

\author{
Martin Clarke, ${ }^{1}$ Conor Duggan, ${ }^{2}$ Clive R. Hollin, ${ }^{3}$ Nick Huband, ${ }^{4}$ Lucy McCarthy, ${ }^{5}$ Steffan Davies ${ }^{6}$
}

The Psychiatrist (2013), 37, 124-129, doi: 10.1192/pb.bp.112.039289

${ }^{1}$ Institute of Mental Health, University of Nottingham; ${ }^{2}$ University of Nottingham and Partnerships in Care; ${ }^{3}$ University of Leicester; ${ }^{4}$ Institute of Mental Health and Nottinghamshire Healthcare NHS Trust:

${ }^{5}$ Nottinghamshire Healthcare NHS Trust; ${ }^{6}$ Countywide Mentally Disordered Offender Services, Northamptonshire Healthcare NHS Trust

Correspondence to Martin Clarke (drmartinclarke@googlemail.com)

First received 8 Mar 2012, final revision 12 Sep 2012, accepted 3 Oct 2012
Aims and method We examined readmission to psychiatric hospital of 550 patients discharged from one medium secure unit over 20 years. Multiple sources were used to obtain readmission data.

Results Readmission was common, particularly to non-secure psychiatric hospitals. At least 339 patients (61.6\%) were readmitted to any psychiatric hospital (mean follow-up 9.5 years), with over a third (37.6\%) subsequently being readmitted to medium- or high-security or both. Of those discharged directly to the community, having previous in-patient treatment and a Mental Health Act classification of mental illness were associated with shorter time to first readmission.

Clinical implications The long-standing nature of disorders is evident in the high rates of readmission overall and the need for readmission to medium and high secure services, suggesting that these patients require long-term follow-up and support from mental health services.

Declaration of interest None.
Outcome studies of patients admitted to medium secure units usually focus on reconviction, ${ }^{1-5}$ but where reported, readmission to any psychiatric hospital was common. ${ }^{6-8}$ The previous largest follow-up of a single unit found that of 234 discharged patients, $174(74 \%)$ were readmitted to a psychiatric hospital (mean follow-up 6.6 years), with more previous admissions and an earlier first psychiatric contact predicting readmission. ${ }^{6}$ Other variables such as age, gender and number of convictions were not associated with readmission. That study also found that 50 former patients (21\%) spent some of the follow-up period in a high secure hospital, including 27 patients discharged directly to such hospitals. ${ }^{6}$ A high rate of readmission to any hospital was also shown in another, albeit smaller, medium secure unit cohort of 63 former patients with schizophrenia, with 56 (89\%) patients requiring at least one readmission to a psychiatric hospital (mean follow-up 5.4 years), of whom 41 (73\%) had multiple readmissions. ${ }^{7}$ A large survey of all medium secure units in England and Wales found a readmission rate of $27 \%$ for women and $21 \%$ for men within 1 year. ${ }^{9}$ Readmission to medium security has also been reported in a follow-up of 70 men with a history of violence or a violent index offence discharged from a medium secure unit to the community (either directly or via lower security). ${ }^{8}$ Followed up for at least 2 years, 33 patients $(47 \%)$ were readmitted to the unit, with 16 patients (23\%) being formally recalled while on restriction orders. Readmission is a proxy measure of both the risk that these patients pose and the continued psychiatric care that they require, so it is an important outcome to assess. Patients in the community in particular will not have all the protection that a secure environment would provide.

This study examined the subsequent readmissions to psychiatric hospitals of patients admitted to Arnold Lodge Medium Secure Unit in Leicester, England, over a 20-year period during which it changed substantially. Overviews of outcomes, mortality and gender differences for this cohort are described elsewhere. ${ }^{10-12} \mathrm{~A}$ description of the first 16 years admission cohort is also available. ${ }^{13}$

\section{Method}

The cohort consisted of 595 first admissions to Arnold Lodge from its opening in July 1983 to 30 June 2003 - a period of 20 years. Four patients died in the unit following their first admission and 41 had not been discharged from their first admission at the census date. Therefore, the study sample comprised all 550 first admissions discharged from Arnold Lodge during the study period.

\section{Data sources}

Admission characteristics were gathered from medical records at Arnold Lodge. Readmission outcome data were obtained from clinical records at Arnold Lodge and other psychiatric services, the Special Hospitals case register and the Home Office Mental Health Unit. Ethical approval was granted by the Trent Multi-centre Research Ethics Committee. The research was conducted under Section 60 of the Health and Social Care Act 2001, allowing for the 
collection of follow-up data without the patient's consent and thereby increasing the completeness of the sample.

\section{Statistical analysis}

Data were analysed using PASW Statistics 18.0 for Windows. Kaplan-Meier survival analyses were conducted to examine time to readmission. The effects of predictor variables on time to readmission were examined using Cox proportional hazards regression analysis. Predictors of readmission were identified using logistic regression analysis. $P$-values less than 0.05 were considered to be statistically significant.

\section{Results}

Of the 550 patients discharged from their first admission to Arnold Lodge, 459 (83.5\%) were men and 91 (16.5\%) women. The majority of patients were White (78.9\%). The median length of stay for this sample was 164 days (range 2-3872) and was longer for women (median 227 days) than for men (median 154 days), although not significantly longer. The mean length of follow-up from discharge to death, loss of contact or the census date was 9.5 years $($ s.d. =4.8). Women had a longer mean follow-up time than men: 11.5 years (s.d.=4.1) v. 9.1 years (s.d.=4.9); $t=4.887$, d.f. $=145.6$, $P<0.001$. There was no significant difference in the mean follow-up times between patients with Mental Health Act 1983 classifications of psychopathic disorder (9.8 years, s.d. $=5.1)$ or mental illness $(9.4$ years, s.d. $=4.6)$. There were 5246 person-years of follow-up after discharge.

Characteristics of the cohort are shown in Table 1. Twothirds of patients had a Mental Health Act classification of mental illness, whereas a quarter had a Mental Health Act classification of psychopathic disorder (Arnold Lodge had a dedicated personality disorder service throughout most of the study period). Men and women had different background characteristics, with $94 \%$ of the men and only $65 \%$ of the women having an index offence. Women (42\%) were also more likely than men (11\%) to be admitted under a civil section of the Act. Almost half the sample (45\%) were discharged to another psychiatric hospital. These comprise transfers to higher security (7\%), transfers to equivalent medium security (3\%) and discharges to lower security, principally non-secure psychiatric hospitals (35\%).

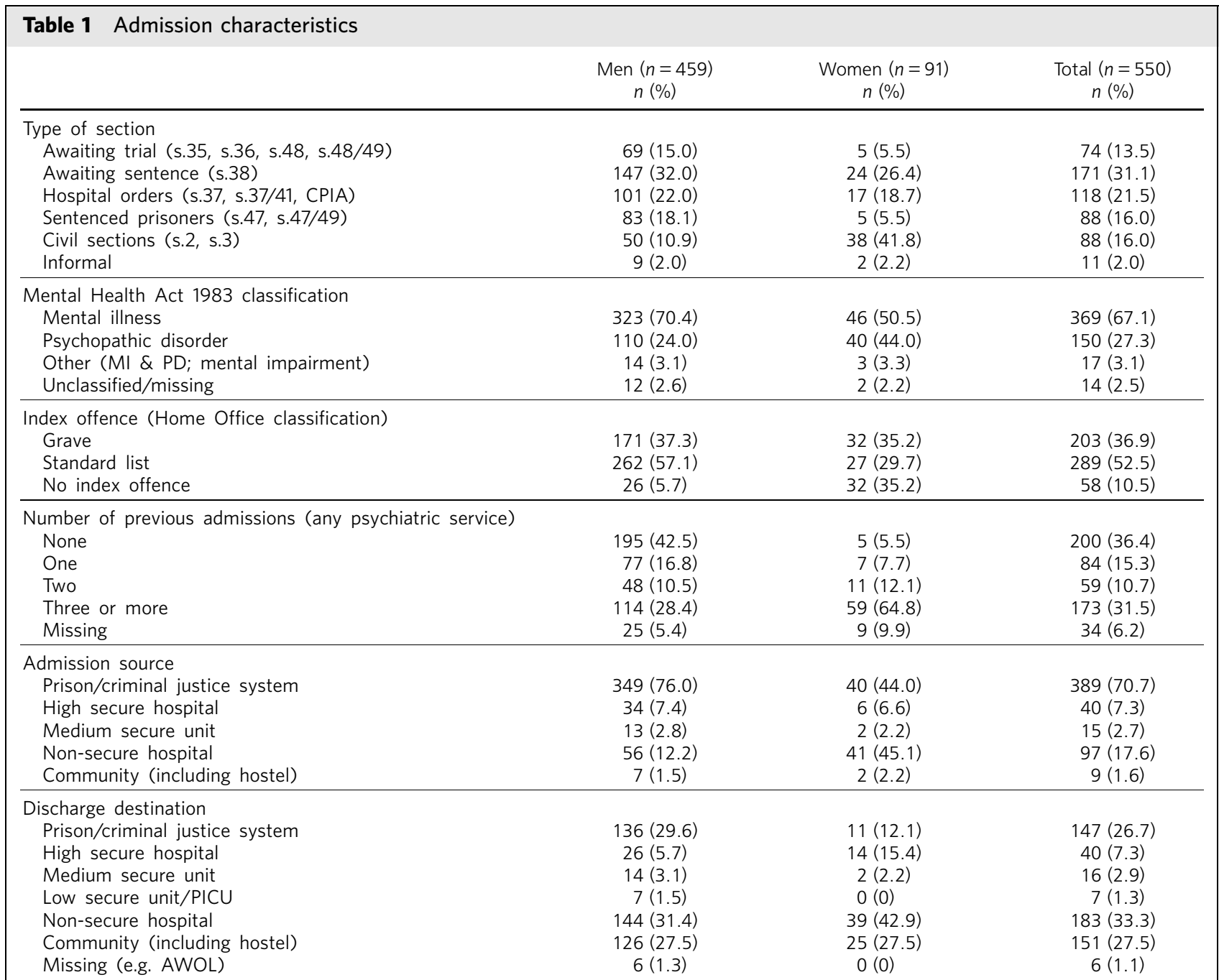

AWOL, absent without leave; CPIA, Criminal Procedure and Investigations Act 1996; MI \& PD, mental illness and psychopathic disorder; PICU, psychiatric intensive care unit. 


\section{Overview of readmission}

In spite of obtaining Section 60 support, there were still considerable problems in accessing data in certain trusts. The main trusts comprised by Arnold Lodge's catchment area (Derbyshire, Leicestershire, Lincolnshire and Nottinghamshire) were very helpful in facilitating the study. Of the 490 patients for whom readmission data were available, 339 patients $(69.2 \%)$ were readmitted to a psychiatric hospital at some point prior to the census. At least 239 patients were known to have been readmitted under provisions of the Mental Health Act, of whom 86 also had at least one informal readmission. An additional 86 patients were informally readmitted at least once to a psychiatric hospital and were not readmitted under provisions of the Act. Over the whole study period there were missing readmission data for 60 patients (10.9\%). Those with missing readmission data had a shorter length of stay (median 111 days) than those whose readmission data were available (median 170 days; $U=11794.0, Z=-2.501$, $P=0.012$ ).

Significantly more women (87.5\%) than men $(65.6 \%)$ were readmitted to hospital $\left(\chi^{2}(1, n=490)=15.05, P<0.001\right.$; odds ratio (OR) $3.67,95 \%$ CI $1.83-7.34$, missing 60 cases). Readmission data were available for 462 patients with a Mental Health Act classification of either psychopathic disorder or mental illness. Of these readmissions, 320 patients $(69.3 \%)$ were readmitted to a psychiatric hospital. Significantly more patients with a classification of mental illness $(72.7 \%)$ than with a classification of psychopathic disorder $(61.0 \%)$ were readmitted $\left(\chi^{2}(1, n=462)=6.14\right.$, $P<0.05$; OR $=1.70,95 \%$ CI 1.12-2.59, missing 57 cases). Readmission frequency data were available for 485 patients (Table 2). In total, 33 patients were readmitted on at least 10 occasions, of whom 12 were readmitted at least 20 times.

\section{Readmission of patients discharged to the community}

More than a quarter of men and women were discharged directly to the community: 126 men (27.5\%) and 25 women (27.5\%). Of these, a higher percentage of women $(79.2 \%)$ than men $(66.7 \%)$ were readmitted to a psychiatric hospital, but this was not significantly higher: $\chi^{2}(1, n=141)=1.45$, $P>0.05$ (missing data for 10 cases). Thirty-one patients (29 men and 2 women) were discharged to the community on a restriction order. Of these, 21 patients (68\%) were readmitted at some point during the follow-up, 13 patients $(42 \%)$ were readmitted informally at least once while on a restriction order and 11 patients $(35 \%)$ were recalled to hospital. Of the 151 patients discharged directly to the community, 40 (26\%) were readmitted to a medium secure unit during the follow-up; these comprised 30 men (24\%) and 10 women (40\%). Eight men (6\%) were admitted to a high secure hospital at some point following their discharge to the community; five of these were also readmitted to a medium secure unit and the other three men were admitted to a high secure hospital as a result of a recall to hospital $(n=1)$ and new offences for which the former patient received a manslaughter conviction $(n=2)$.

Time to readmission for those discharged to the community was first examined using Kaplan-Meier survival analysis, with cases censored on death or at the census at 30 June 2003. Potential predictors of time to readmission were expressed as binary variables (either directly or via median splits): gender; ethnicity (White $v$. non-White); Mental Health Act classification of mental illness; grave index offence; custodial sentence prior to age 18 years; problematic behaviour at school; contact with child mental health services; alleged childhood sexual abuse; alleged childhood physical abuse; previous in-patient care; previous self-harm; previous attempted suicide; history of 'severe' alcohol misuse; history of 'severe' drug misuse; age at admission; number of previous convictions; age at first conviction; length of stay; ${ }^{14}$ and discharge on a restriction order. Only two of these variables (previous in-patient care and Mental Health Act classification of mental illness) had a significant effect on the survival rates. The log rank test (Mantel-Cox) indicated that the survival time to the first readmission for those discharged directly to the community was significantly shorter for patients with a classification of mental illness than for patients with a classification of psychopathic disorder: $\chi^{2}(1, n=141)=8.1, \quad P<0.01$. The median time to first readmission for patients with a classification of mental illness was 1.9 years compared with 5.2 years for those with a classification of psychopathic disorder. The survival time to the first readmission for those discharged directly to the community was significantly shorter for people who had received previous in-patient care: $\chi^{2}(1, n=140)=10.7, \quad P<0.01$. The median times to first readmission for patients who had received previous in-patient care and those who had not were 2.0 years and

\begin{tabular}{l} 
Table 2 Number of readmissions categorised by gender and Mental Health Act classification \\
\cline { 2 - 7 } \\
\cline { 2 - 7 }
\end{tabular}




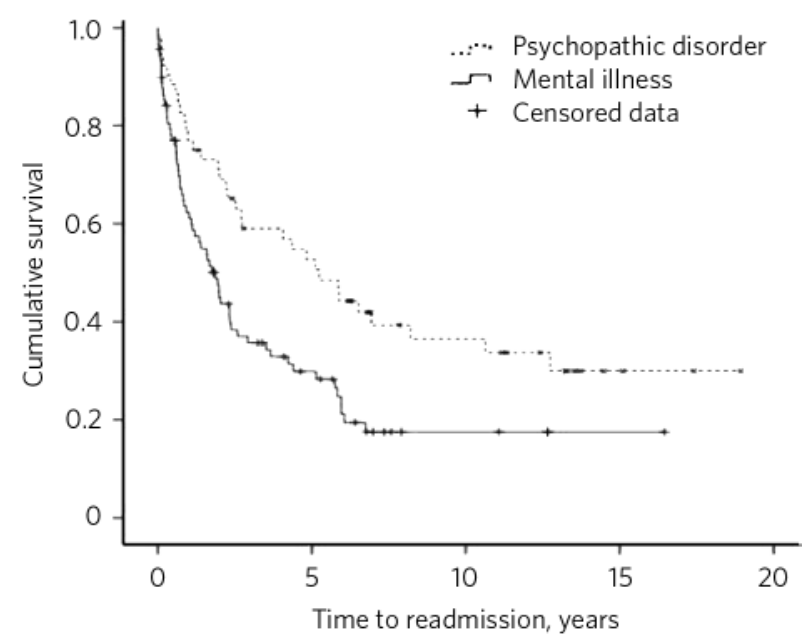

Fig 1 Kaplan-Meier curve for readmission of patients discharged directly to the community according to their Mental Health Act classification.

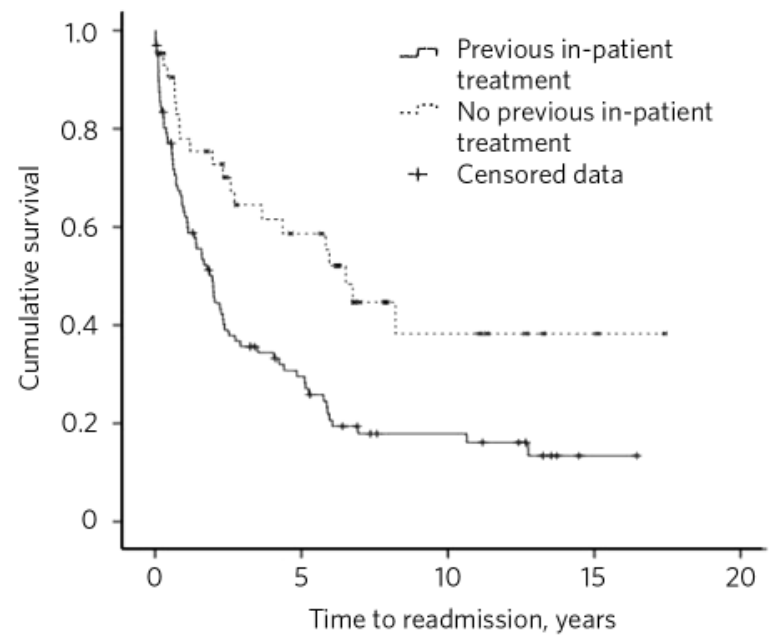

Fig 2 Kaplan-Meier curve for readmission of patients discharged directly to the community according to previous in-patient care.

6.5 years respectively. Survival curves for these variables are shown in Figs 1 and 2.

A Cox proportional hazards model, which allows for the effects of several predictor variables, was then developed to investigate the time to readmission for patients discharged directly to the community by taking into account the significant predictors. This model examined the time to readmission controlling for previous in-patient care and Mental Health Act classification of mental illness or psychopathic disorder (Table 3). Patients who had received previous in-patient treatment had a hazard of readmission 2.2 times higher than those who had not. Also, patients with a classification of mental illness had a hazard of readmission 1.8 times higher than those with psychopathic disorder. The model was not significantly improved by adding interactions between the predictor variables.

A logistic regression analysis was then conducted to examine which of the 19 variables predicted readmission ( $n=112$, missing data 39). Prediction success overall was $80 \%(94 \%$ for readmission but only $50 \%$ for no readmission). Two variables made a significant contribution to prediction: previous in-patient treatment and not having a history of self-harm. Those with previous in-patient treatment were 5.7 times more likely to be readmitted, and a history of self-harm was associated with an $88 \%$ decrease in the odds of being readmitted. Three variables approached significance: classification of mental illness $(P=0.055)$, number of previous convictions $(P=0.055)$ and age $(P=0.050)$.

\section{Readmission to Arnold Lodge and other secure hospitals}

Of the 550 discharged patients, 113 (20.5\%) were readmitted to Arnold Lodge. Of these, 19 (3.5\%) were readmitted twice and $7(1.3 \%)$ were readmitted three times. There was no significant difference in gender ( $19.6 \%$ men, $25.3 \%$ women) or in Mental Health Act classification (22.2\% mental illness, $16.7 \%$ psychopathic disorder) in readmission to Arnold Lodge. A further 43 patients (7.8\%) - excluding patients who were discharged directly to another medium secure unit were readmitted to other medium secure units, resulting in 156 patients (28.4\%) being readmitted to medium secure care at some point during the follow-up. Forty-two patients were admitted to a high secure hospital (40 transferred directly) at some point during the follow-up, resulting in 82 patients (14.9\%) spending time in high secure care after discharge from Arnold Lodge. Twelve patients (2.2\%) had two admissions to a high secure hospital. Experiencing further secure care following discharge was common, with 207 patients $(37.6 \%)$ subsequently spending some time in medium and/or high security.

\section{Time of readmission}

In the first year after discharge, 121 patients $(22.0 \%)$ had at least one readmission to a psychiatric hospital. This group comprised 92 men (20.0\%) and 29 women (31.9\%). The overall percentage rises to $31.6 \%$ when those who spent all their first year in hospital $(n=101)$ or had missing data $(n=66)$ are excluded.

Table 3 Estimates of variables for Cox regression (for readmission)

\begin{tabular}{lcccccc} 
& & & & & \\
& B & s.e. & Wald & d.f. & $P$ & Hazard ratio $(95 \%$ Cl) \\
\hline Previous in-patient & 0.784 & 0.248 & 9.991 & 1 & 0.002 & $2.190(1.347-3.561)$ \\
\hline Mental illness & 0.577 & 0.219 & 6.936 & 1 & 0.008 & $1.780(1.159-2.734)$ \\
\hline
\end{tabular}


Time spent in institutions

Some patients remained 'institutionalised' in hospital or prison throughout the follow-up. Data were available for 535 discharged patients, of whom $46(8.6 \%)$ remained in a hospital throughout the follow-up. The mean time in hospital after transfer from Arnold Lodge for these 46 patients was 6.3 years $($ s.d. $=4.7)$. When time spent in prison was included, 88 (16.5\%) of the 534 discharged patients for whom data were available remained either in hospital or in prison throughout the follow-up. The mean time in hospital or prison after discharge for these patients was 5.7 years (s.d. = 4.6).

\section{Discussion}

Readmission was common for patients discharged from their first admission to Arnold Lodge medium secure unit (62\%), particularly to non-secure psychiatric hospitals. Women were 3.7 times more likely than men to be readmitted. Many patients had several readmissions over the follow-up period - an outcome noted in a different medium secure unit sample. ${ }^{7}$ Readmission is not of itself a negative outcome; for example, informal readmission to an open psychiatric unit is a far better outcome than the need to admit to high security following a serious offence. Over a quarter of patients were readmitted at least once to a medium secure unit - a fifth to Arnold Lodge - and this proportion would have been larger if another unit had not opened that readmitted patients from the north of the region. Over a third of those discharged from their first admission to Arnold Lodge subsequently spent time in medium or high security, with some patients spending time in both. Over a quarter were discharged directly to the community from their first admission; nonetheless, a quarter of these were readmitted to a medium secure unit during the follow-up.

Of those discharged directly to the community, only previous in-patient treatment and having a mental illness were associated with shorter time to first readmission. Previous in-patient treatment was also a predictor of readmission to any hospital. In addition, a history of selfharm was associated with a decreased risk of readmission. This may be explained by self-harm being more common in those with a classification of psychopathic disorder compared with mental illness, a predictor that approached significance. It is clinically intuitive that the mentally ill group would have more previous and future hospital admissions. Indeed, in our cohort two-thirds of men with a Mental Health Act classification of mental illness had a previous admission compared with less than half of men with a classification of psychopathic disorder (further information available from authors). Conversely, patients with a classification of psychopathic disorder had a shorter time to reconviction than those with mental illness. ${ }^{14}$ Incidentally, the risk of death for patients with a classification of either mental illness or psychopathic disorder was higher than that for the general population. However, although the risk of death was higher for patients with mental illness compared with psychopathic disorder, the difference was not significant. ${ }^{11}$
Using the same exclusion criteria (missing data and those who spent all their first year in hospital), the overall percentage of patients readmitted at least once to a psychiatric hospital in the first year after discharge (32\%) was similar to that of a national survey of medium secure units (28\%). ${ }^{9}$ The percentage of patients readmitted to any psychiatric hospital (69\%) approaches that found in the previous largest follow-up of a single medium secure unit (74\%). ${ }^{6}$ However, our study had a longer mean follow-up: 9.5 years $v$. 6.6 years. A smaller percentage of discharged patients $(15 \%)$ spent some of the follow-up period in a high secure hospital than that found by Maden et al (21\%). ${ }^{6}$ These rates of readmission have to be placed in a national context as they will have been affected by the contraction in the numbers of high secure beds and the expansion of medium and low secure units. Community treatment has also undergone substantial changes, for example, assertive outreach, community forensic teams, crisis resolution and home treatment services now provide treatment in the community that was not previously available. The legal powers available for community treatment also changed during the study period, ${ }^{15}$ with the introduction of supervised discharge in 1996 (superseded by supervised community treatment orders in the 2007 amendments to the Mental Health Act). The patients' diagnoses also have implications as to whether services are likely to accept them. Arnold Lodge has a dedicated personality disorder unit and consequently this cohort has more cases of personality disorder than reported in other medium secure unit studies.

Multiple sources were used to corroborate information and to minimise attrition. Missing data for readmission to any psychiatric hospital were fairly low (11\%) given the large sample size and long follow-up period. The research was conducted under Section 60 of the Health and Social Care Act 2001. Despite this, delays imposed by one trust made it impractical to collect data from their case files and made contacting further trusts pointless; this accounts for some missing readmission data. Further limitations were that data were collected retrospectively and that the study is of a single unit; therefore the findings may not be generalisable to other medium secure units.

\section{Study implications}

Patients discharged from medium security have long-term conditions and present long-term risks requiring support from mental health services. Our cohort comprised a group of men and women who were at risk of reconviction of a new offence, ${ }^{10}$ premature death, particularly by suicide, ${ }^{11}$ and readmission to secure psychiatric services. All these adverse outcomes are most common in the year following discharge, but the risk remains over many years of followup. Those treated in medium security remain at risk of recurrence of their mental disorder and risk events for many years and require careful long-term follow-up, retaining a detailed knowledge of their conditions and risks - a difficult task in modern services with multiple teams and transitions in care. 


\section{Funding}

The initial description of the cohort and follow-up was funded by Nottinghamshire Healthcare National Health Service (NHS) Trust; further follow-up was funded by the NHS National Forensic Mental Health Research and Development Programme.

\section{About the authors}

Martin Clarke (PhD) is a Research Fellow at the Institute of Mental Health University of Nottingham, UK; Conor Duggan (PhD, MD, FRCPsych) is Emeritus Professor Forensic Mental Health at the Institute of Mental Health, University of Nottingham, UK and Head of Research and Development for Partnerships in Care; Clive R. Hollin (PhD) is Professor of Criminological Psychology in the School of Psychology at the University of Leicester, UK; Nick Huband (PhD) is a Clinical Research Fellow, Section of Forensic Mental Health at the Institute of Mental Health, University of Nottingham, and Nottinghamshire Healthcare NHS Trust, UK; Lucy McCarthy $(\mathrm{PhD})$ is a Senior Research Fellow at Arnold Lodge, Nottinghamshire Healthcare NHS Trust, Leicester, UK; Steffan Davies (FRCPsych) is Consultant Forensic Psychiatrist at Northamptonshire Healthcare NHS Foundation Trust, Northampton, UK.

\section{References}

1 Friendship C, McClintock T, Rutter S, Maden A. Re-offending: patients discharged from a Regional Secure Unit. Crim Behav Ment Health 1999; 9: $226-36$.

2 Edwards J, Steed P, Murray K. Clinical and forensic outcome 2 years and 5 years after admission to a medium secure unit. J Forensic Psychiatry 2002; 13: 68-87.

3 Falla S, Sugarman P, Roberts L. Reconviction after discharge from a regional secure unit. Med Sci Law 2000; 40: 156-7.

4 Maden A, Scott F, Burnett R, Lewis GH, Skapinakis P. Offending in psychiatric patients after discharge from medium secure units: prospective national cohort study. BMJ 2004; 328: 1534.
5 Gray NS, Snowden RJ, MacCulloch S, Phillips H, Taylor J, MacCulloch MJ. Relative efficacy of criminological, clinical, and personality measures of future risk of offending in mentally disordered offenders: a comparative study of HCR-20, PCL:SV, and OGRS. J Consult Clin Psychol 2004; 72: 523-30.

6 Maden A, Rutter S, McClintock T, Friendship C, Gunn J. Outcome of admission to a medium secure psychiatric unit. I. Short- and long-term outcome. Br J Psychiatry 1999; 175: 313-6.

7 Baxter R, Rabe-Hesketh S, Parrott J. Characteristics, needs and reoffending in a group of patients with schizophrenia formerly treated in medium security. J Forensic Psychiatry 1999; 10: 69-83.

8 Dolan M, Khawaja A. The HCR-20 and post-discharge outcome in male patients discharged from medium security in the UK. Aggress Behav 2004; 30: 469-83.

9 Maden A, Skapinakis P, Lewis G, Scott F, Burnett R, Jamieson E. Gender differences in reoffending after discharge from medium-secure units. National cohort study in England and Wales. Br J Psychiatry 2006; 189 168-72.

10 Davies S, Clarke M, Hollin C, Duggan C. Long-term outcomes after discharge from medium secure care: a cause for concern. Br J Psychiatry 2007; 191: 70-4.

11 Clarke M, Davies S, Hollin C, Duggan C. Long-term suicide risk in forensic psychiatric patients. Arch Suicide Res 2011; 15: 16-28.

12 Sahota S, Davies S, Duggan C, Clarke M, Huband N, Owen V. Women admitted to medium secure care: their admission characteristics and outcome as compared with men. Int J Forensic Ment Health 2010; 9: 110-7.

13 Ricketts D, Carnell H, Davies S, Kaul A, Duggan C. First admissions to a regional secure unit over a 16 -year period: changes in demographic and service characteristics. J Forensic Psychiatry 2001; 12: 78-89.

14 Gibbon S, Huband N, Bujkiewicz S, Hollin C, Clarke M, Davies S, et al. The influence of admission characteristics on outcome: evidence from a medium secure forensic cohort. Personal Ment Health 2013; 7: 1-10.

15 Davies S. Compulsory treatment in the community: current legal powers. Adv Psychiatr Treat 2002; 8: 180-8. 